\title{
Infrared Spectroscopy of Jupiter and Saturn
}

\author{
Pierre Drossart \\ DESPA, Observatoire de Paris, section de Meudon, France
}

\begin{abstract}
The spectroscopy of giant planets in the infrared range gives access to a remote sensing of many physical parameters. The composition, pressure/temperature structure, and the cloud structure all contribute to the spectrum, in solar reflected light below 3 micrometer as well as thermal emission above, from atmospheric levels ranging from the mesosphere down to the troposphere. Imaging spectroscopy revealing the variability of the atmosphere gives access to spatial and temporal evolution of these parameters, constraining the meteorological evolution of the planets.
\end{abstract}

Infrared spectroscopy is a powerful tool for studying the atmosphere of giant planets, as the best compositional diagnostic for molecular emissions, as well as an atmospheric sounder from the deeper tropospheric levels (pressures of 5 to 8 bars at $5 \mu \mathrm{m}$ for Saturn and Jupiter respectively) to the thermosphere at microbar levels. The infrared range also covers several emission mechanisms from the solar reflected component, modified by molecular planetary absorptions in the near infrared $(1-3 \mu \mathrm{m})$ to the thermal emission component above 3 micron, with in each case different atmospheric modulations by cloud particles and thermal profiles.

The thermal profiles of Jupiter and Saturn delimitate several atmospheric regions:

- the thermosphere, in the uppermost layers, exhibit in both planets a strong heating by magnetospheric precipitating particles and solar UV absorption.

- the stratosphere (1 $\mu \mathrm{bar}-300 \mathrm{mbar})$ is the region of deposition for most of the solar energy, exhibits almost an isothermal structure. It is mainly controlled by methane thermal emission through the strong bands of $\nu_{4}$, and absorption in the main fundamentals and overtone vibrational bands.

- the troposphere, where most of the meteorological motions take place is the region of condensation of the water clouds, which play an important role in the heat transport of the atmosphere.

In the stratospheres of Saturn and Jupiter, methane is the strongest radiator and controls the thermal regulation of these planets by radiating internal and external heat fluxes. The main molecular emission and absorption observed in giant planets are due to the following compounds, which have long been studied in the laboratory to measure accurate line lists necessary for radiative transfer calculations:

- $\mathrm{CH}_{4}$ (Wenger \& Champion 1998) 
- $\mathrm{NH}_{3}$ (Kleiner et al. 1999)

- $\mathrm{PH}_{3}$ (Tarrago et al. 1996)

- $\mathrm{CH}_{3} \mathrm{D}, \mathrm{AsH}_{3}, \mathrm{GeH}_{4}, \mathrm{H}_{2} \mathrm{O}$, etc. (retrieved from Hitran or Geisa databank)

Despite a low abundance of a few ppm, phosphine $\left(\mathrm{PH}_{3}\right)$ has strong molecular signatures at $5 \mu \mathrm{m}$, a window of high atmospheric transparencies, which gives strong absorption, dominating the spectra of Jupiter and Saturn at 5 micron. A good knowledge of the molecular absorption in this spectral range has allowed the retrieval of faint $\mathrm{H}_{2} \mathrm{O}$ absorptions, due to tropospheric water vapor, in the Jovian and Saturnian atmospheres (Encrenaz et al. 1996).

Radiative transfer models start from an atmospheric structure, molecular spectroscopic data base to provide synthetic spectra of the planets with a good degree of accuracy above roughly 2 micron; on the contrary, in the near infrared, below 2 micron, the lack of accurate spectroscopy presently limits the accuracy of the modeling. Models are usually limited to band models, where average molecular absorption in wavelength intervals are used instead of more exact line by line calculations. The frontier separating the two domains is continuously regressing toward shorter wavelengths and constitutes one of the major challenge for molecular spectroscopy devoted to atmospheric research.

The difficulties of radiative transfer are exemplified in the region of the $\nu_{4}$ band of methane at $7.8 \mu \mathrm{m}$ where high resolution spectra exhibit numerous very narrow lines, with widths of the order of $0.002 \mathrm{~cm}^{-1}$ with a saturated core at a brightness temperature corresponding to a stratospheric temperature. At low spectral resolution, the simple structure of the Jovian spectra masks in fact the very high complexity of the original calculations, needed for accurate measurements of the thermal structure.

Due to the high accuracy reached by synthetic calculations, it is today possible to detect faint molecular features, and to interpret them. A recent example is the detection of $\mathrm{CH}_{3} \mathrm{CH}_{2}$ and $\mathrm{C}_{2} \mathrm{H}_{4}$ in the atmosphere of Saturn (Fouchet et al. 2000). Another example is the detection of tropospheric $\mathrm{H}_{2} \mathrm{O}$ in the atmosphere of Saturn at 5 micron, where faint $\mathrm{H}_{2} \mathrm{O}$ absorption lines are identified against a very complex spectrum dominated by $\mathrm{PH}_{3}$ absorption, well interpreted through radiative transfer modeling.

A more difficult problem is to take into account in line by line calculations the accurate line shapes of molecular absorption or emission. Numerous complex quantum effects can affect the line shapes, like the asymmetry of lines, departing from Voigt profiles, collisional interferences, line mixing effects, etc. These effects are only poorly known in laboratory today, and their implications on the planetary modeling begin only to be identified (Pieroni et al. 2001).

Molecular abundances in the stratosphere are governed by photochemical equilibrium. The photochemical effects in a hydrogenated atmosphere include very complex reactions, and only the main lines of the equations are known, with some difficulties to reach quantitative agreement with the observations (Moses et al. 2000). An example of recent improvements in this direction is the detection of $\mathrm{CH}_{3}$ on Saturn, and the interpretation of its abundance (Bézard et al. 1998).

Most of the calculations for radiative transfer in the giant planet atmospheres are made at local thermodynamic equilibrium. A remarkable example of non-LTE interpretations has been the detection of $\mathrm{CH}_{4}$ fluorescence on Jupiter 
(Drossart et al. 1999). This detection gives a sounding of the upper atmospheric levels responsible for important absorption and emission mechanisms, and still poorly known presently.

The need for accurate photochemical and recombination rates is also very high for planetary atmospheres modeling. An example of a long awaited parameter is the recombination rate of $\mathrm{H}_{3}^{+}$, which governs the ionospheric chemistry, but is still controversial despite many years of laboratory and interpretation works. Other items on the list of the most wanted coefficients are the vibrational relaxation rates of methane, used in non LTE methane emissions models.

At the end of the process, the communication of the laboratory work must be included in accessible databases for use by the planetary community. Several databases exist: Hitran, Geisa, etc. which must still be completed and developed to reach always higher accuracy and completion. This is the necessary steps for higher accuracy in planetary atmospheric works, which will be needed for the ongoing studies devoted to Saturn in the context of the Cassini mission.

\section{References}

Bézard, B., Feuchtgruber, H., Moses, J., \& Encrenaz, T. 1998, A\&A, 334, L41

Drossart, P., Fouchet, T., Crovisier, J., Lellouch, E., Encrenaz, T., Feuchtgruber, H., \& Champion, J.P. 1999, in The Universe as seen by ISO, ESA SP427, Eds. P. Cox and M.F. Kessler, 169

Encrenaz, T., Drossart, P., Feuchtgruber, H., Fouchet, T., \& Atreya, S.K. 1996, Planet. Space Sci., 47, 1225

Fouchet, T., Lellouch, E., Bézard, B., Feuchtgruber, H., Drossart, P., \& Encrenaz, T. 2000, A\&A, 355, L13

Kleiner, I., Brown, L.R., Tarrago, G., Kou, Q.L., Picqué, N., Guelachvili, G., Dana, V., \& Mandin, J.Y. 1999, J. Molec. Spectrosc., 193, 46

Lellouch, E., Bézard, B., Fouchet, T., Lellouch, E., Bézard, B., Feuchtgruber, H., Drossart, P., \& Encrenaz, T. 2000, A\&A, 355, L13

Moses, J., Lellouch, E., Bézard, B., Gladstone, G.R., Feuchtgruber, H., \& Allen, M. 2000, Icarus, 145,16

Pieroni, D., Hartmann, J.-M., Camy-Peyret, C., Jeseck, P., \& Payan, S. 2001, J. Quant. Spectrosc. Rad. Transfer, 68, 117

Tarrago, G., Lacome, N., Levy, A., Guleachvili, G., Bézard, B., \& Drossart, P. J. 1992, Molec. Spectrosc., 154, 30

Wenger, Ch. \& Champion, J.P. 1998, J. Quant. Spectrosc. Rad. Transfer, 59, 471 